\title{
Fractal vector measures and vector calculus on planar fractal domains
}

\author{
F. Mendivil \\ Department of Mathematics and Statistics \\ Acadia University \\ Wolfville, Nova Scotia, Canada B0P 1X0 \\ franklin.mendivil@acadiau.ca \\ E.R. Vrscay \\ Department of Applied Mathematics \\ University of Waterloo \\ Waterloo, Ontario, Canada N2L 3G1 \\ ervrscay@links. uwaterloo.ca
}

February 25, 2002

\begin{abstract}
We define an abstract framework for self-similar vector-valued Borel measures on a compact space $X$ based upon a formulation of Iterated Function Systems on such measures. This IFS method permits the construction of tangent and normal vector measures to planar fractal curves. Line integrals of smooth vector fields over planar fractal curves may then be defined. These line integrals then lead to a formulation of Green's Theorem and the Divergence Theorem for planar regions bounded by fractal curves. The abstract setting also naturally leads to "probability measure"-valued measures. These measures may be used to color the geometric attractor of an IFS in a self-similar way.
\end{abstract}

\section{Introduction}

Since the classical exposition on fractal geometry by Benoit Mandelbrot [20], fractal analysis has received much attention in the physical and biological sciences because of its potential use in modelling and understanding "real world" phenomena - for example, systems that exhibit disorder or irregularity. There is overwhelming experimental evidence that various materials, (e.g. diffusion limited aggregates) demonstrate, over a limited range of scales, geometric selfsimilarities that influence both static (e.g. electronic band structure) as well as dynamic (e.g. diffusion) properties of these materials. (Since it is impossible to 
provide an even partial list of the important developments in these areas, we simply refer the more general reader to an interesting collection of papers [6].)

Much attention in the literature has been given to the problem of transport near and across irregular boundaries. Such transport behaviour is important in electrochemical cells and electrodeposition in general, involving the diffusion of materials and their adsorption onto the electrodes. Mathematically, the problem is to solve Laplace's equation in the electrolyte with boundary conditions that reflect the electrochemical processes at the electrode surface. Various studies have employed fractal boundaries to simulate the self-similarity of such irregular electrodes over a finite range of scales. Some studies have been experimental, involving electrodes that have been constructed to assume dendritic, fractal-like structures (over macroscopic scales) [19]. Other studies have been numerical, involving Monte Carlo/random walk simulations over fractal-like structures [23]. These works have led to approaches that use scaling arguments to derive the nonlinear response of irregular electrodes in terms of exponents that involve their fractal dimensions [21, 13]. (Indeed, the latter approach has stimulated interesting work on the formulation of vector difference calculus - including Maxwell and Navier-Stokes equations - over discrete lattices [24].) It is also important to mention the work of Evertesz and Mandelbrot [8] in which equipotential lines associated with charge distributions over self-similar trees were computed. (Indeed, the idea of "equilibrium charge distributions" associated with invariant measures can be traced to early work on Julia-Fatou theory of iteration of rational functions [5].)

We have been inspired by the above studies and, in particular, by the recent work of M. Giona [13], to develop a method of constructing vector fields over fractal curves that could be useful in the study of phenomena that involve irregular boundaries/regions, such as the transport problems listed above. As Giona pointed out, "a general mathematical physics on fractal curves, encompassing the generalization of standard vector calculus (e.g. Stokes, Gauss, Green theorems), has not yet been developed, however, mostly because of the impossibility of establishing a local (i.e. pointwise) definition of the notion of tangent and normal vectors." Our method is a generalization of the method of Iterated Function Systems (IFS) developed originally by Hutchinson [18] in a geometric measure theoretic setting and later analyzed by Barnsley and Demko [2] (who gave the name "IFS") from a probabilistic viewpoint. IFS and its various generalizations have been useful for the construction of fractal sets and measures as well as for the actual analysis of sets, measures and functions. In particular, IFS has provided the basis of "fractal image compression" [11].

Indeed, Giona [13] developed an effective heuristic IFS-type method of defining and computing line integrals of smooth vector fields over fractal curve attractors of IFS. He presented strong numerical evidence suggesting that tangent and normal vector measures could be defined over such fractal curves (in terms of convergent sequences of appropriate line integrals) as well as conjecturing the existence of Divergence and Green's Theorems over regions enclosed by fractal boundary curves. In this paper we provide a general mathematical framework for such an IFS-based construction of vector fields - a method of IFS over vector- 
valued measures, to be conveniently abbreviated as IFSVVM. We show that the IFSVVM provides a method of constructing and analyzing a variety of vector fields over domains. By appropriate choices of parameters, these IFSVVM vector field attractors can exhibit either "fractal" or "non-fractal" characteristics. In addition, we show how integrals of these vector measures with smooth vector fields can be computed numerically in a recursive manner. We illustrate our method with some simple examples. Our intention is to apply this method to more "realistic" physical problems in the future, including those identified by Giona in the latter part of his paper. Indeed, we hope that this method may be applicable to other interesting problems involving irregular domains, for example, the vibration of "fractal drums" [22]. Another interesting result of our IFS-type method is a random iteration algorithm or "chaos game" that can be used to render IFS attractors with vector values, essentially "coloring" them.

For the benefit of the general reader, let us summarize the most important features of IFS. Let $(X, d)$ be a complete metric space and let $\mathcal{H}(X)$ denote the set of non-empty compact subsets of $X$. The metric space $(\mathcal{H}(X), h)$, where $h$ denotes the Hausdorff metric, is complete [9]. Now let $w_{i}: X \rightarrow X, 1 \leq$ $i \leq N$, be a set of contraction mappings with contraction factors $s_{i} \in[0,1)$. Associated with this Iterated Function System $\mathbf{w}$ is the set-valued IFS operator $\hat{\mathbf{w}}: \mathcal{H}(X) \rightarrow \mathcal{H}(X)$ defined as follows:

$$
\hat{\mathbf{w}}(S)=\bigcup_{i} \hat{w}_{i}(S), \quad \forall S \in \mathcal{H}(X)
$$

where $\hat{w}_{i}(S)=\left\{w_{i}(x): x \in S\right\}$. Then [18]

$$
h(\hat{\mathbf{w}}(A), \hat{\mathbf{w}}(B)) \leq \operatorname{sh}(A, B) \quad \forall A, B \in \mathcal{H}(X),
$$

where $s=\max _{i}\left\{s_{i}\right\}<1$, i.e. $\hat{\mathbf{w}}$ is a contraction mapping. Therefore, from Banach's Fixed Point Theorem, there exists a unique element $A \in \mathcal{H}(X)$ such that $A=\hat{\mathbf{w}}(A)$, referred to as the attractor of the IFS $\mathbf{w}$.

Now let $\mathbf{p}=\left\{p_{1}, p_{2}, \ldots, p_{N}\right\}$ denote a set of probabilities associated with the IFS maps, $\sum_{i} p_{i}=1$. Let $\mathcal{M}_{1}(X)$ denote the space of probability measures on the Borel sigma field of $X$ with Monge-Kantorovich metric $d_{M}$ (more details in Section 2). Associated with this IFS with probabilities (w, p) is a "Markov" operator $M: \mathcal{M}_{1} \rightarrow \mathcal{M}_{1}$ defined as follows. For a $\mu \in \mathcal{M}_{1}$ :

$$
(M \mu)(S)=\sum_{i} p_{i} \mu\left(w_{i}^{-1}(S)\right), \quad \forall S \subset \mathcal{H}(X) .
$$

Then [18]

$$
d_{M}(M \mu, M \nu) \leq s d_{M}(\mu, \nu), \quad \forall \mu, \nu \in \mathcal{M}_{1}(X) .
$$

Therefore, there exists a unique measure $\bar{\mu} \in \mathcal{M}_{1}(X)$ such that $\bar{\mu}=M \bar{\mu}$, referred to as the invariant measure of the $\operatorname{IFSP}(\mathbf{w}, \mathbf{p})$.

In order to understand the method of [13], consider an IFS with a nonintersecting fractal curve $C$ as its attractor. Let $A$ and $B$ be the endpoints of $C$. Repeated action of the IFS upon the line segment $\overline{A B}$ produces a sequence 
of segmented curves $C_{i}$ that converge in Hausdorff metric to $C$. This procedure mimics the classical construction of fractal curves using "generators," as illustrated nicely in Mandelbrot's book [20].

Given a (smooth) vector field $f(x)$, Giona, in the "spirit of calculus," then examined the line integrals $\int_{C_{i}} f(x) \cdot \hat{T} d s$ where $\hat{T}$ denotes the unit tangent vector. Since each segment of $C_{i+1}$ is obtained by the action of a particular IFS map on curve $C_{i}$, these line integrals can be computed recursively using a method of "IFS with Orientations," keeping track of the rotations $R_{i}$ associated with each of the contractive geometric IFS maps $w_{i}$. (In [13], the IFS maps $w_{i}$ were similitudes and shared a common contraction factor.) Numerical calculations with several examples suggested that the line integrals over the approximations $C_{i}$ converged to a limit which was then defined to be the line integral over the fractal curve attractor $C$. As in the case of IFSP, integrals over IFS fractal curve attractors with integrands of the form $x_{1}^{m} x_{2}^{n}$ can be computed in a recursive manner. Giona then applied this method to compute line integrals over closed fractal curves. Numerical calculations supported the idea of a Green's Theorem over regions enclosed by fractal curves.

The structure of this paper is as follows. In Section 2 we introduce a MongeKantorovich type metric on vector-valued measures. In Section 3, we define the Iterated Function System on vector-valued measures (IFSVVM). This construction is used to define self-similar measure-valued measures in Section 4, a generalization of IFS with probabilities. This apparatus allows the construction of a "chaos game" to color fractal IFS attractors. In Section 5, the IFSVVM method is used to construct tangent and normal vector measures to fractal curves in the plane. This allows a definition of line integrals of smooth vector fields over such curves. These results are then used in Section 6 to develop Green's Theorem and the Divergence Theorem over regions with (nonintersecting) fractal curve boundaries.

The formulation of vector calculus over regions enclosed by fractal curves has been studied by J. Harrison and coworkers [16, 17]. Their methods are very different from ours in that they use an extension of Whitney's geometric integration theory. This theory defines integrals on limits of formal sums of geometric objects by a very clever choice of norm of these formal sums of geometric objects. In effect, a fractal object is represented by a convergent series of geometric objects. In contrast, we are constructing explicit vector measures on the fractal curves themselves. Line integrals of sufficiently smooth vector fields can then be defined as bounded linear functionals on an appropriate function space.

Finally, we mention that during the final preparation of this manuscript, we discovered two recent papers along similar themes [10, 3]. In [10], the authors develop a similar method to construct vector-valued measures. The basis of their method is a general measure-theoretic construction in which a measure is produced by means of a repeated subdivision which is performed in a consistent manner. The authors also apply this method to construct a vector-valued measure that is "tangent" to a curve and discuss the case of self-similar vector measures. However, our construction of self-similar vector-valued measures 
differs in that it is based upon an IFS-type framework in which convergence is determined by contractivity. As a result, convergence to the limiting measure is guaranteed for a large class of initial measures.

In [3] the authors consider Markov operators on spaces of vector-valued measures. However, they restrict their study to the case of operators which satisfy a boundedness condition which our operators, in general, do not. Their operator is very similar to our IFS operator (Eq. (7)) but the boundedness restriction does not allow the application to vector calculus.

\section{Metric on vector-valued measures}

Let $(X, d)$ be a compact metric space and let $\mathcal{M}\left(X, \mathbb{R}^{n}\right)$ be the set of vectorvalued measures on the Borel sigma field of $X$ with values in $\mathbb{R}^{n}$. Let $\mathcal{L} i p\left(X, \mathbb{R}^{n}\right)$ be the set of Lipschitz functions $f: X \rightarrow \mathbb{R}^{n}$ such that $\|f(x)-f(y)\| \leq K d(x, y)$ for some $K \geq 0$ and $\mathcal{L} i p_{1}\left(X, \mathbb{R}^{n}\right)$ be the subset of $\mathcal{L} i p\left(X, \mathbb{R}^{n}\right)$ for which $K=1$. For $\mu, \eta \in \mathcal{M}\left(X, \mathbb{R}^{n}\right)$ we define the Monge-Kantorovich metric

$$
d_{M}(\mu, \eta)=\sup \left\{\int_{X} f(x) \cdot d(\mu-\eta)(x): f \in \mathcal{L} i p_{1}\left(X, \mathbb{R}^{n}\right)\right\} .
$$

It is clear that $d_{M}(\mu, \eta)=d_{M}(\eta, \mu) \geq 0$. Suppose that $\mu, \nu, \eta \in \mathcal{M}\left(X, \mathbb{R}^{n}\right)$. Then (all suprema are taken over $f \in \mathcal{L} i p_{1}\left(X, \mathbb{R}^{n}\right)$ )

$$
\begin{aligned}
\sup \int_{X} f \cdot d(\mu-\eta) & =\sup \int_{X} f \cdot d(\mu-\nu+\nu-\eta) \\
& \leq \sup \int_{X} f \cdot d(\mu-\nu)+\sup \int_{X} f \cdot d(\nu-\eta) .
\end{aligned}
$$

Thus, $d_{M}(\mu, \eta) \leq d_{M}(\mu, \nu)+d_{M}(\nu, \eta)$ (under the assumption that all of these "distances" are finite). In addition, $d_{M}(\mu, \eta)=0$ if and only if $\mu=\eta$. Thus, the only property that $d_{M}(\cdot, \cdot)$ lacks to be a metric is that it is possible for $d(\cdot, \cdot)$ to be infinite.

Example Let $\mu=2 \eta$, then

$$
\int_{X} f(x) \cdot d(\mu-\eta)=\int_{X} f(x) \cdot d(\eta) .
$$

The final integral can be arbitrarily large since, for any $w \in \mathbb{R}^{n}, f+w \in$ $\mathcal{L} i p_{1}\left(X, \mathbb{R}^{n}\right)$ for any $f \in \mathcal{L} i p_{1}\left(X, \mathbb{R}^{n}\right)$.

Let $v \in \mathbb{R}^{n}$ and $C>0$ and consider the affine subspace $\mathcal{S}_{v}\left(X, \mathbb{R}^{n}\right)$ of $\mathcal{M}\left(X, \mathbb{R}^{n}\right)$ defined as

$$
\mathcal{S}_{v}\left(X, \mathbb{R}^{n}\right)=\left\{\mu \in \mathcal{M}\left(X, \mathbb{R}^{n}\right): \mu(X)=v\right\}
$$


For any $\mu, \eta \in \mathcal{S}_{v}\left(X, \mathbb{R}^{n}\right)$ and $f \in \mathcal{L} i p_{1}\left(X, \mathbb{R}^{n}\right)$,

$$
\begin{aligned}
\left|\int_{X} f(x) \cdot d(\mu-\eta)\right| & =\left|\int_{X}\left(f(x)-f\left(x_{0}\right)\right) \cdot d(\mu-\eta)+f\left(x_{0}\right) \cdot \int_{X} d(\mu-\eta)\right| \\
& =\left|\int_{X}\left(f(x)-f\left(x_{0}\right)\right) \cdot d(\mu-\eta)\right| .
\end{aligned}
$$

In order to bound the final integral it is necessary to restrict $\mu$ and $\eta$ to some bounded subset of $\mathcal{S}_{v}\left(X, \mathbb{R}^{n}\right)$. Thus, we will assume that all of the measures we choose are restricted to some subset $S$ of $\mathcal{S}_{v}\left(X, \mathbb{R}^{n}\right)$ such that $\mu \in S$ implies that $\|\mu\| \leq C$ for some $C$, where $\|\mu\|$ is the semivariation of $\mu$ (see [4]). In this case,

$$
\left|\int_{X}\left(f(x)-f\left(x_{0}\right)\right) \cdot d(\mu-\eta)\right| \leq 2 C \operatorname{diam}(X)
$$

since $f \in \mathcal{L i p}_{1}\left(X, \mathbb{R}^{n}\right)$. We therefore define

$$
\mathcal{S}_{v}^{c}\left(X, \mathbb{R}^{n}\right)=\left\{\mu \in \mathcal{M}\left(X, \mathbb{R}^{n}\right): \mu(X)=v,\|\mu\| \leq C\right\} .
$$

This set is weak* compact, being a closed bounded subset of vector measures. Since $d_{M}(\cdot, \cdot)$ is bounded on $\mathcal{S}_{v}^{c}\left(X, \mathbb{R}^{n}\right)$, it is a metric. Since $\mathcal{S}_{v}^{c}\left(X, \mathbb{R}^{n}\right)$ is compact, it is complete under this metric. (Notice that $d_{M}(\cdot, \cdot)$ induces the weak* topology on $\mathcal{M}\left(X, \mathbb{R}^{n}\right)$.)

\section{$3 \quad$ IFS on vector-valued measures}

Let $w_{i}$ be contractive IFS maps on $X$ and $p_{i} \geq 0$ be associated real numbers. We now relax the restriction that $\sum_{i} p_{i}=1$. Further, let $R_{i}$ be linear operators on $\mathbb{R}^{n}$. We define the operator $T: \mathcal{M}\left(X, \mathbb{R}^{n}\right) \rightarrow \mathcal{M}\left(X, \mathbb{R}^{n}\right)$ associated with this IFSVVM $(\mathbf{w}, \mathbf{p}, \mathbf{R})$ as

$$
T(\mu)(B)=\sum_{i} p_{i} R_{i} \mu\left(w_{i}^{-1}(B)\right)
$$

for all Borel sets $B \subset X$. If we impose the condition

$$
\left(\sum_{i} p_{i} R_{i}\right) v=v
$$

for some vector $v \in \mathbb{R}^{n}$ then, for any $\mu \in \mathcal{S}_{v}\left(X, \mathbb{R}^{n}\right)$,

$$
\begin{aligned}
T(\mu)(X) & =\sum_{i} p_{i} R_{i} \mu\left(w_{i}^{-1}(X)\right) \\
& =\sum_{i} p_{i} R_{i} v \\
& =v .
\end{aligned}
$$

Thus the two conditions for $T: \mathcal{S}_{v}\left(X, \mathbb{R}^{n}\right) \rightarrow \mathcal{S}_{v}\left(X, \mathbb{R}^{n}\right)$ become 
C1: $\left(\sum_{i} p_{i} R_{i}\right) v=v$

C2: $\mu(X)=v$ and $\|\mu\| \leq C$ implies that $\|T(\mu)\| \leq C$.

Proposition 1 Let $T$ be the IFS operator defined above and suppose that $T$ maps $\mathcal{S}_{v}^{c}\left(X, \mathbb{R}^{n}\right)$ to itself for some $v \in \mathbb{R}^{n}$ and $C>0$. Then

$$
d_{M}(T(\mu), T(\eta)) \leq\left(\sum_{i} s_{i} p_{i}\left\|R_{i}^{t}\right\|\right) d_{M}(\mu, \eta)
$$

for all $\mu, \eta \in \mathcal{S}_{v}\left(X, \mathbb{R}^{n}\right)$.

Proof: $\quad$ Let $f \in \mathcal{L} i p_{1}\left(X, \mathbb{R}^{n}\right)$. Then, for $\mu$ and $\eta$ in $\mathcal{M}\left(X, \mathbb{R}^{n}\right)$,

$$
\begin{aligned}
\left.\int_{X} f(x) \cdot d_{M}(T(\mu)-T(\eta))\right) d x & =\sum_{i} p_{i} \int_{X} f(x) \cdot d\left(R_{i}\left(\mu\left(w_{i}^{-1}(x)\right)-\eta\left(w_{i}^{-1}(x)\right)\right)\right) \\
& =\sum_{i} p_{i} \int_{X} f\left(w_{i}(y)\right)^{t} R_{i} d(\mu(y)-\eta(y)) \\
& =\int_{X}\left(\sum_{i} p_{i}\left(R_{i}^{t} f\left(w_{i}(y)\right)\right)\right)^{t} d(\mu(x)-\eta(x)) \\
& =\left(\sum_{i} p_{i} s_{i}\left\|R_{i}^{t}\right\|\right) \int_{X} \phi(y)^{t} d(\mu(y)-\eta(y))
\end{aligned}
$$

where $\phi(y)=\frac{1}{\sum_{i} p_{i} s_{i}\left\|R_{i}^{t}\right\|} \sum_{i} p_{i} R_{i}^{t} f\left(w_{i}(x)\right) \in \mathcal{L} i p_{1}\left(X, \mathbb{R}^{n}\right)$, since $s_{i}$ is the Lipschitz factor of $w_{i}$. (Details below.) Taking the supremum, we obtain

$$
d_{M}(T(\mu), T(\eta)) \leq\left(\sum_{i} p_{i} s_{i}\left\|R_{i}^{t}\right\|\right) d(\mu, \eta)
$$

and the result follows.

For completeness, we now show that $\phi(x) \in \mathcal{L} i p_{1}\left(X, \mathbb{R}^{n}\right)$. Let $x, y \in X$ and $f \in \mathcal{L} i p_{1}\left(X, \mathbb{R}^{n}\right)$ and $s=\sum_{i} s_{i} p_{i}\left\|R_{i}^{t}\right\|$. Then

$$
\begin{aligned}
\|\phi(x)-\phi(y)\| & =\left\|s^{-1} \sum_{i} p_{i} R_{i}^{t}\left(f\left(w_{i}(x)\right)-f\left(w_{i}(y)\right)\right)\right\| \\
& \leq s^{-1} \sum_{i} p_{i}\left\|R_{i}^{t}\right\|\left\|f\left(w_{i}(x)\right)-f\left(w_{i}(y)\right)\right\| \\
& \leq s^{-1} \sum_{i} p_{i}\left\|R_{i}^{t}\right\| d\left(w_{i}(x), w_{i}(y)\right) \\
& \leq s^{-1} \sum_{i} p_{i} s_{i}\left\|R_{i}^{t}\right\| d(x, y) \\
& \leq d(x, y) .
\end{aligned}
$$


If $T: \mathcal{S}_{v}\left(X, \mathbb{R}^{n}\right) \rightarrow \mathcal{S}_{v}\left(X, \mathbb{R}^{n}\right)$ and $0 \neq w \in \mathbb{R}^{n}$, we can easily modify $T$ to obtain a new operator $\hat{T}: \mathcal{S}_{w}\left(X, \mathbb{R}^{n}\right) \rightarrow \mathcal{S}_{w}\left(X, \mathbb{R}^{n}\right)$. Let $R$ be any rotation such that $R(v)=(\|v\| /\|w\|) w$. Then

$$
\begin{aligned}
\hat{T}(\mu)(B) & =\frac{\|w\|}{\|v\|} R\left(\sum_{i} p_{i} R_{i} R^{-1} \mu\left(w_{i}^{-1}(B)\right)\right) \\
& =\frac{\|w\|}{\|v\|} \sum_{i} p_{i}\left(R \circ R_{i} \circ R^{-1}\right) \mu\left(w_{i}^{-1}(B)\right)
\end{aligned}
$$

has the desired property. Simply stated, we modify $\hat{R}_{i}=R \circ R_{i} \circ R^{-1}$ and $\hat{p}_{i}=\|w\| /\|v\| p_{i}$.

The case $n=2$ is special. If $\sum_{i} p_{i} R_{i} v=v$ for some $v \in \mathbb{R}^{2}$, then, in fact, $\sum_{i} p_{i} R_{i} w=w$ for all $w \in \mathbb{R}^{2}$. To show this, let $R$ be a rotation so that $R(v)=w$ (with no loss in generality, $\|v\|=\|w\|)$. Then

$$
\left(\sum_{i} p_{i} R_{i}\right) w=\sum_{i} p_{i} R_{i} R v=R\left(\sum_{i} p_{i} R_{i} v\right)=R v=w .
$$

Thus we have the following condition on the $p_{i}$ and the rotations $R_{i}$ :

$$
\sum_{i} p_{i} R_{i}=I
$$

the identity matrix. In this case, the functional forms of $T$ and $\hat{T}$ are identical - the only difference between $T$ and $\hat{T}$ are their domains and ranges. This property will be used in Section 5. The operator that constructs the tangent vector measure of a fractal curve $C$ will be modified appropriately in order to construct the normal vector measure of $C$.

We close this section by stating the simple, yet important, consequence of Proposition 1 above.

Proposition 2 Let $T$ be the IFS operator defined in Eq. (7) such that conditions $C 1$ and $C 2$ are satisfied for a given vector $v$. Furthermore assume that

$$
c=\sum_{i} s_{i} p_{i}\left\|R_{i}^{t}\right\|<1 .
$$

Then there exists a unique measure $\mu \in \mathcal{S}_{v}\left(X, \mathbb{R}^{n}\right)$ such that $T \mu=\mu$. Furthermore $\operatorname{supp}(\mu)=A$, the attractor of the $\operatorname{IFS} \mathbf{w}$.

\section{Self-Similar Measure-valued Measures}

The first application of the general IFSVVM method described above will be made to to the case of measure-valued measures. In some sense, this is the true 
generalization of the standard method of IFS with probabilities (IFSP) over the set set $\mathcal{M}_{1}(X)$ of Borel probability measures on $X$ (cf. Section 1 ). Note that the positivity of any measure $\mu \in \mathcal{M}_{1}(X)$ along with the normalization condition $\mu(X)=1$ imply that $\mu$ is bounded in the variation norm.

As motivation, let us begin with a variation of the standard random iteration algorithm or "Chaos Game" [1] for rendering the attractor of an IFS with probabilities. (This variation was told to the authors by J. Anderson.) Given a set of $N$ IFS maps $w_{i}$ with probabilities $p_{i}, \sum_{i} p_{i}=1$, the standard chaos game algorithm is as follows:

1. Pick an initial point $x_{0}$ on the IFS attractor. One way to do this is to let $x_{0}$ be the fixed point of $w_{1}$. Set $n=0$.

2. With probabilities defined by the $p_{i}$ 's, choose one of the $w_{i}$ 's, say $w_{j}$.

3. Let $x_{n+1}=w_{j}\left(x_{n}\right)$. Plot $x_{n+1}$.

4. If a sufficient number of points $x_{n}$ have been generated, stop. Otherwise, let $n \rightarrow n+1$ and go to Step 2 .

The variation consists of adding "color" to the algorithm. Assign a color $C_{i}$ to each map $w_{i}$ and and a probability $0 \leq p c_{i} \leq 1$ of changing to this color. Then, the new algorithm is as follows:

1. Pick $x_{0}$ to be the fixed point of $w_{1}$ and set the current color to $c_{1}=C_{1}$. Set $n=0$.

2. Choose a map $w_{j}$ according to the probabilities $p_{i}$.

3. Change the current color to color $C_{j}$ with probability $p c_{j}$. That is, with probability $p c_{j}$ set $c_{n+1}=C_{j}$ else $c_{n+1}=c_{n}$.

4. Set $x_{n+1}=w_{j}\left(x_{n}\right)$ and plot $x_{n+1}$ using color $c_{n+1}$.

5. If a sufficient number of points have been generated, stop. Otherwise, let $n \rightarrow n+1$ and go to Step 2 .

This algorithm yields a plot of the attractor "colored" in a self-similar way by the colors associated with the IFS maps $w_{i}$. Figure 1 is an illustration of such a colored version of the Sierpinski triangle (a 3-map IFS) that was generated with $p c_{i}=1 / 2$ for all $i$. Since a black-and-white copy of the attractor will obviously not show the true coloring, we provide a very brief description. Let $w_{i}, i=1,2,3$, denote the IFS maps for this attractor with fixed points $(0,0)$, $(1,0)$ and $(0,1)$, respectively. Associated with these maps are the colors red, green and blue, respectively. As one travels toward a vertex, the color of the attractor points approaches the color associated with the IFS map with that vertex as fixed point. For example, as one approaches $(1,0)$, the color of the attractor points becomes more green. 


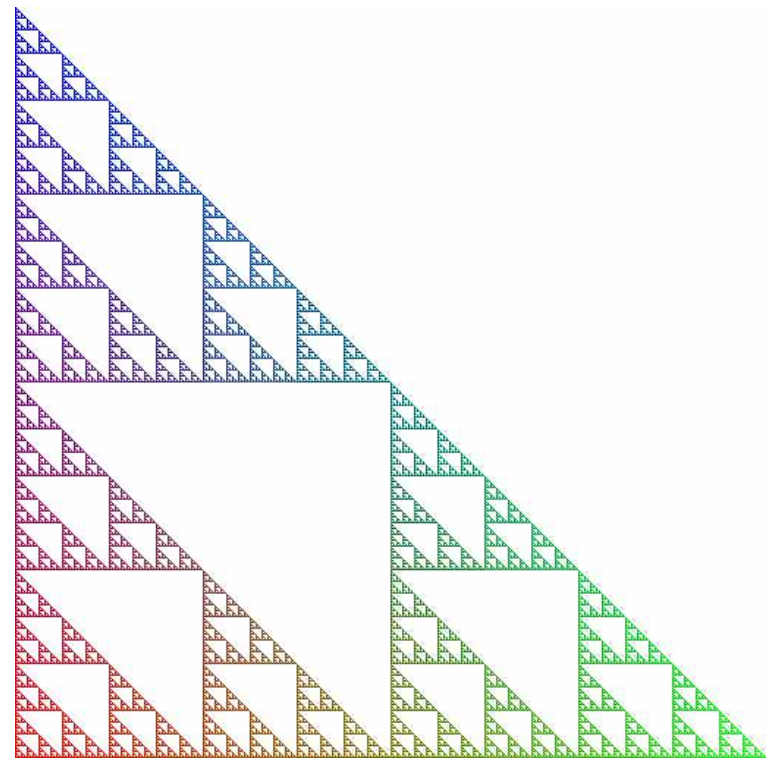

Figure 1: Sierpinski Triangle colored by the "color chaos game"

We now present an IFS operator that can generate a "colored" attractor as its fixed point. Let $\Lambda$ be a finite set with $n=|\Lambda|$ and $M_{i}$ be Markov transition matrices on $\Lambda$. Since we wish to multiply the $M_{i}$ 's by probability vectors on the right, we require them to be column stochastic, that is, the column sum of each matrix $M_{i}$ is 1 .

For our set of $N$ IFS maps $w_{i}$ with probabilities $p_{i}, \sum_{i} p_{1}=1$, we define the "color Markov" operator $M_{C}: \mathcal{M}\left(X, \mathbb{R}^{n}\right) \rightarrow \mathcal{M}\left(X, \mathbb{R}^{n}\right)$ by

$$
M_{C}(\mu)(B)=\sum_{i} p_{i} M_{i} \mu\left(w_{i}^{-1}(B)\right)
$$

for all Borel sets $B \subset X$. In other words, we use Markov transition matrices for the linear operators that define $M$. Now let $v$ be an invariant measure for the stochastic matrix $\sum_{i} p_{i} M_{i}$. (Being a convex combination of column stochastic matrices, this matrix is column stochastic.) The set we use to replace $\mathcal{S}_{v}^{c}\left(X, \mathbb{R}^{n}\right)$ from Section 2 is the space

$$
\mathcal{P}\left(X, \mathbb{R}^{n}\right)=\left\{\mu \in \mathcal{M}\left(X, \mathbb{R}^{n}\right): \mu(B) \geq 0, \forall B \subset X, \mu(X)=v\right\} .
$$

Notice that this set is automatically bounded in semivariation (and in total variation as well). Clearly $M_{C}$ maps $\mathcal{P}\left(X, \mathbb{R}^{n}\right)$ to itself. The condition that the $M_{i}$ be stochastic implies that $\left\|M_{C}(\mu)\right\| \leq C$ if $\|\mu\| \leq C$. Also note that if $A$ is a Markovian matrix then $\|A\| \geq 1$. 
Proposition 3 Let $M_{C}$ be the IFS operator defined above on $\mathcal{P}\left(X, \mathbb{R}^{n}\right)$. Then

$$
{ }_{M}\left(M_{C}(\mu), M_{C}(\eta)\right) \leq\left(\sum_{i} s_{i} p_{i}\left\|M_{i}^{t}\right\|\right) d_{M}(\mu, \eta)
$$

for all $\mu, \eta \in \mathcal{P}\left(X, \mathbb{R}^{n}\right)$.

Now assume that $M_{C}$ is contractive on $\mathcal{P}\left(X, \mathbb{R}^{n}\right)$ and let $\vec{\mu} \in \mathcal{P}\left(X, \mathbb{R}^{n}\right)$ be the invariant measure for $M_{C}$. Then if $|\eta|$ denotes the total variation of $\eta$, we have $|\vec{\mu}|(B)=|M(\vec{\mu})|(B)$. Denoting $\mu=|\vec{\mu}|$, we have that $\mu$ is the attractor of the $\operatorname{IFSP}(\mathbf{w}, \mathbf{p})$ :

$$
\mu(B)=\sum_{i} p_{i} \mu\left(w_{i}^{-1}(B)\right) .
$$

Heuristically, we can think of $\vec{\mu}$ as

$$
d \vec{\mu}(x)=\vec{f}(x) d \mu(x)
$$

where $\vec{f}(x)$ is the "attractor" of the "IFS"

$$
\vec{f}(x) \longrightarrow \sum_{i} M_{i} \vec{f}\left(w_{i}^{-1}(x)\right) .
$$

Since $\vec{\mu}$ is absolutely continuous with respect to $\mu$, we know that $\vec{f}(x)$ exists and is the Radon-Nikodym derivative of $\vec{\mu}$ with respect to $\mu$.

The matrices $M_{i}$ which correspond to the "chaos game with color" are

$$
M_{i}=\left(1-p c_{i}\right) I+p c_{i} J_{i}
$$

where

$$
J_{i}=\left(\begin{array}{ccccc}
0 & 0 & 0 & \cdots & 0 \\
0 & 0 & 0 & \cdots & 0 \\
\vdots & \vdots & \vdots & \ddots & \vdots \\
1 & 1 & 1 & \cdots & 1 \\
\vdots & \vdots & \vdots & \ddots & \vdots \\
0 & 0 & 0 & \cdots & 0 \\
0 & 0 & 0 & \cdots & 0
\end{array}\right)
$$

and the $i^{\text {th }}$ row is a row of ones. It is easy to see that $\left\|M_{i}\right\|_{1}=1$, so that the contractivity condition becomes

$$
\sum_{i} s_{i} p_{i}<1
$$

For the Sierpinski Triangle example with equal probabilities, $s_{i}=p_{i}=\frac{1}{2}$ so that this sum is $\frac{3}{4}$. 
The connection between the color chaos game and the IFS operator $M_{C}$ is simple. Given a set $B \subset X$ with $\mu(B)>0$, the probability measure on $\Lambda$ defined by

$$
\frac{\vec{\mu}(B)}{\mu(B)}
$$

describes the distribution of the colors on the set $B$. If $B$ represents a pixel on the computer screen, then this distribution describes the percentage of time that this pixel is each color over the run of the chaos game.

Note that $J_{i} w=e_{i}$ for any probability vector $w$ (where $e_{i}$ is the $i$ th basis vector in $\left.\mathbb{R}^{n}\right)$. So, if $p c_{i}=p c$ for all $i$ and letting $\vec{p}=\left(p_{1}, p_{2}, \ldots, p_{N}\right)$, we see that

$$
\left(\sum_{i} p_{i} M_{i}\right) \vec{p}=(1-p c) \vec{p}+p c \vec{p}=\vec{p}
$$

so that $\vec{p}$ is the invariant distribution for $\sum_{i} p_{i} M_{i}$. Therefore $\vec{\mu}(X)=\vec{p}$. The interpretation is as follows. If probability of changing to any color is the same for all colors, then the distribution of color content of the entire image is just proportioned by the $p_{i}$ 's. This is not to say that $\vec{\mu}$ will be the same regardless of the value of $p c$. In the limiting case that $p c=1$, each subtile of the attractor will be colored only by its own color.

In closing this chapter, we point out that chaos games for IFS-type operators over function spaces have also been devised [12].

\section{$5 \quad$ Application to fractal curves}

In this section, we apply the general IFSVVM framework to the construction of vector measures defined on fractal curves. The motivation is simple and best illustrated with an example. Consider the classical construction of the von Koch fractal curve under repeated applications of the generator $G$ illustrated in Figure 2.

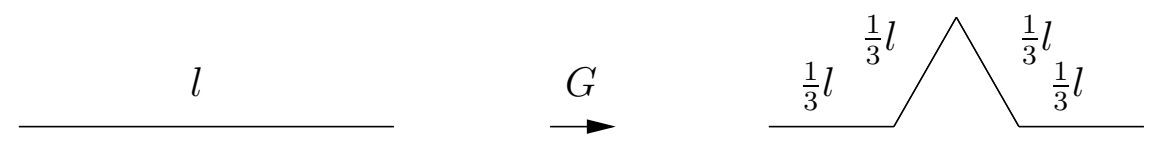

Figure 2: Generator of the von Koch fractal curve

If we begin with the seed curve $C_{1}=[0,1]$ on the $x$-axis then the sequence of curves $C_{n+1}=G\left(C_{n}\right)$ converges in Hausdorff metric to the von Koch curve shown in Figure 3.

Of course, the action of the generator is accomplished geometrically by the application of an appropriate four-map affine IFS w. (The four maps $w_{i}$ are given in the Appendix.) Now, in addition to the geometric construction illustrated above, let us construct a sequence of tangent measures to the curves $C_{i}$ in an appropriate IFS-type fashion. Suppose that we begin with a "uniform" 


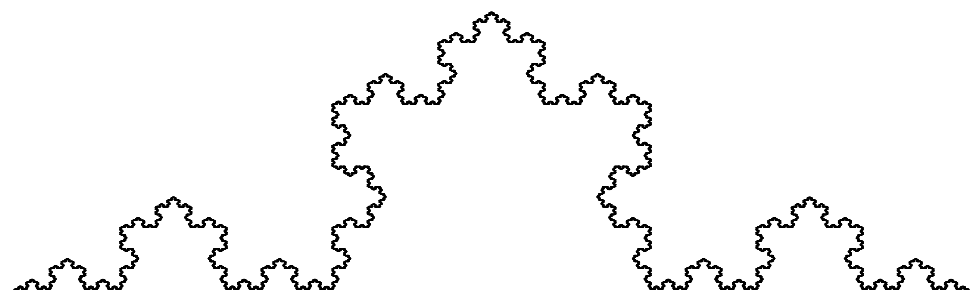

Figure 3: The von Koch fractal curve

vector measure $v$ on $C_{1}$ which can be interpreted as the tangent vector to $C_{1}$. We now produce four contracted and rotated copies of $v$ and place them appropriately onto the segments that comprise $C_{2}$. This process is illustrated in Figure 4.

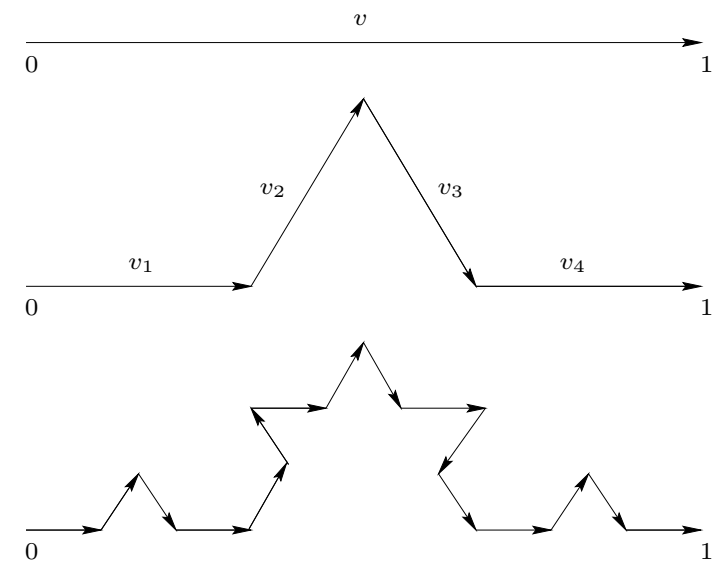

Figure 4: First three iterations of the tangent measure for the von Koch curve

In general, let $C$ be a (continuous) curve that is the attractor of an affine $N$-map IFS w. Let $A$ and $B$ denote the endpoints of $C, L$ the line segment $\overline{A B}$ and $v$ the vector $\overrightarrow{A B}$. Then the image of $L$ under the IFS will be a piecewise linear approximation to the curve. Let $v_{i}=w_{i}(v)$. Then $\sum_{i} v_{i}=v$ (since the attractor of the IFS is a continuous curve, the head of $v_{i-1}$ must touch the tail of $v_{i}$ ). Let $R_{i}$ be the rotation that takes $v$ into the direction of $v_{i}$ and 
$p_{i}=\left\|v_{i}\right\| /\|v\|$. Then $p_{i} R_{i}(v)=v_{i}$ so we have

$$
\left(\sum_{i} p_{i} R_{i}\right) v=\sum_{i} v_{i}=v .
$$

Define the IFS operator $T$ (as in equation (7)) using the above $R_{i}$ and $p_{i}$. Let us assume that $T$ is a contraction on $\mathcal{S}_{v}^{c}\left(X, \mathbb{R}^{n}\right)$ so that has a unique fixed point $\mu \in \mathcal{S}_{v}^{c}\left(X, \mathbb{R}^{n}\right)$, i.e. $T \mu=\mu$. Then the sequence $T^{n}(\eta)$ converges to $\mu$ for any $\eta \in \mathcal{S}_{v}^{c}\left(X, \mathbb{R}^{n}\right)$. A convenient choice of initial measure $\eta \in \mathcal{S}_{v}^{c}\left(X, \mathbb{R}^{n}\right)$ is as follows: Choose any probability measure $\lambda$ on $X$ and define

$$
\eta(B)=v \lambda(B),
$$

for all Borel sets $B \in X$, where $v$ is the displacement vector defined above. Clearly $\eta(X)=v \lambda(X)=v$ so that $\eta \in \mathcal{S}_{v}\left(X, \mathbb{R}^{n}\right)$. Thus, $T^{n}(\eta) \rightarrow \mu$ as $n \rightarrow \infty$.

With the above construction of $T$ and our choice of $v=\overrightarrow{A B}, \mu$ may be interpreted as the tangent vector measure to $C$. To see why, let let $\lambda$ be the normalized arclength measure on $L$. If we define $\eta$ as $\eta(B)=v \lambda(B)$, then $\eta$ is the "tangent vector measure" to $L$. The iteration of $T$ produces piecewise linear curves $C_{i}=\mathbf{w}^{n}(L)$ with tangent measures $\mu_{n}=T^{n}(\eta)$. Since the curves $C_{i}$ converge to $C$ and the $\mu_{n}$ converge to $\mu$, one may interpret $\mu$ as the tangent vector measure to $C$.

One might think of $\mu$ heuristically as $d \mu(s)=\vec{T}(s) d s$, where $T(s)$ is the "unit tangent" at $s$ and $d s$ is the arclength measure. We emphasize that this is not the case since $C$ typically has no tangent vector anywhere. However, the analogy with smooth curves is useful. Furthermore, there are some striking similarities. If $B \subset C$ is some segment of our fractal curve $C$, then

$$
\int_{B} d \mu=\mu(B)=B_{\text {end }}-B_{\text {initial }}
$$

is the vector which is the "displacement" from the initial point of $B$ to the final point of $B$. This corresponds to the case of a smooth curve $C^{\prime}$ where $d \mu$ is equal to $\vec{T}(s) d s$ so

$$
\int_{B} d \mu=\int_{B} \vec{T}(s) d s=B_{\text {final }}-B_{\text {initial }} .
$$

Now let us consider the special case of planar curves $C$ in $\mathbb{R}^{2}$. The appropriate IFS operator $T$ is defined as above. In order to to generate the unit normal vector measure to $C$, we simply insert an additional rotation $R$ by $\pi / 2$ to produce the IFS operator $\hat{T}$ from Section 2. $\hat{T}$ maps the initial tangent vector $v$ onto the vector $w$ in the direction outward from the curve. The attractor measure for $\hat{T}$ will then be the normal vector measure to $C$. As mentioned earlier, $T=\hat{T}$ (except that the domains and ranges of $T$ and $\hat{T}$ are different, so they can have different fixed points).

In general, however, the operator $T$ is not necessarily contractive. As an example, consider the Koch curve, the construction of which was illustrated in 
Figure 4 . Let $\mu_{0}$ be the tangent measure to the initial line segment and $\mu_{n}$ be the tangent measure to the piecewise curve obtained on the $n^{\text {th }}$ iteration. In this case $s_{i}=p_{i}=1 / 3$ so that $\sum_{i} s_{i} p_{i}=4 / 9<1$. However, it is easy to show that

$$
\left\|\mu_{n}\right\|=\left\lfloor 4^{n} / 3\right\rfloor\left(2 / 3^{n}\right)+\left(1 / 3^{n}\right)
$$

which clearly shows that $\left\|\mu_{n}\right\| \rightarrow \infty$. Thus, we can find no $C>0$ so that if $\|\nu\|<C$ then $\|T \nu\|<C$ as well. The basic reason for this is that the Koch curve has infinite length.

It is therefore necessary to prove convergence of the measures $\mu_{n}$ in some other way. Convergence may be shown by using duality and interpreting the limiting object as a measure, however not a measure in the usual sense. At best this "measure" will be finitely additive and defined only on an algebra of sets (not closed under countable unions).

Notice that $T^{*}$ defined by

$$
T^{*}(f)(x)=\sum_{i} p_{i} R_{i}^{*} f\left(w_{i}(x)\right)
$$

is the dual operator to $T$. $T^{*}$ maps a continuous function $f$ to another continuous function. Now suppose that $f \in \mathcal{L} i p\left(X, \mathbb{R}^{n}\right)$ with Lipschitz factor $l$ and that $s=\sum_{i} s_{i} p_{i}<1$. Then for any $x, y \in X$ we have

$$
\begin{aligned}
\left\|T^{*} f(x)-T^{*} f(y)\right\| & =\left\|\sum_{i} p_{i} R_{i}\left(f\left(w_{i}(x)\right)-f\left(w_{i}(y)\right)\right)\right\| \\
& \leq \sum_{i} p_{i}\left\|f\left(w_{i}(x)\right)-f\left(w_{i}(y)\right)\right\| \\
& \leq \sum_{i} p_{i} l s_{i} d(x, y) \\
& =\operatorname{sld}(x, y) .
\end{aligned}
$$

Therefore the the Lipschitz factor of $T^{*} f$ is less than $l$. Consequently, the limit of the sequence of functions $\left(T^{*}\right)^{n} f$ is a constant function. Now define $\mathcal{T}_{v}: \mathcal{L} \operatorname{ip}\left(X, \mathbb{R}^{n}\right) \rightarrow \mathbb{R}$ by

$$
\mathcal{T}_{v}(f)=\left(\lim _{n \rightarrow \infty}\left(T^{*}\right)^{n}(f)(x)\right) \cdot v .
$$

If $\mu_{0}$ is uniform vector measure with $\mu_{0}(X)=v$ then

$$
\left(\sum_{i} p_{i} R_{i}^{*} \int_{X} f\left(w_{i}(x)\right) d x\right) \cdot v=\int_{X} T^{*} f(x) d \mu_{0}=\int_{X} f(x) d\left(T\left(\mu_{0}\right)\right) .
$$

If we denote by $\mu$ the "limiting measure" we have

$$
\mathcal{T}_{v}(f)=\int_{X} \lim _{n \rightarrow \infty}\left(T^{*}\right)^{n} f d \mu_{0}=\int_{X} f d \mu .
$$

This indicates that this definition of $\mathcal{T}$ is consistent for it to be dual to $\mu$. 
Using this dual formulation, we can define "limiting measure" $\mu$ as a generalized vector measure in the sense that integrals can be defined with respect to it. However, only functions in $\mathcal{L} i p\left(X, \mathbb{R}^{n}\right)$ can be integrated with respect to $\mu$ since the integral of an arbitrary continuous vector-valued function on $X$ may be unbounded. In other words, $\mathcal{T}$ is an unbounded linear functional on $C\left(X, \mathbb{R}^{n}\right)$ (the set of continuous $\mathbb{R}^{n}$-valued functions on $X$ ) but a bounded linear functional on $\mathcal{L} i p\left(X, \mathbb{R}^{n}\right)$. The corresponding "measure" is at most a finitely additive vector measure of unbounded semivariation defined on an algebra of sets.

It is not necessary to choose $R_{i}$ and $p_{i}$ in the manner indicated above. The only condition necessary for convergence is that $T: \mathcal{S}_{v}\left(X, \mathbb{R}^{2}\right) \rightarrow \mathcal{S}_{v}\left(X, \mathbb{R}^{2}\right)$ for some $v \in \mathbb{R}^{2}$ (along with the contraction condition, of course). Thus, we could choose any other fractal curve $C^{\prime}$ with same initial and final points as $C$, and same number of maps in the IFS as $C$, and use the $R_{i}$ and $p_{i}$ derived from $C^{\prime}$. However, clearly the invariant measure $\mu$ so obtained will not be the tangent or normal vector measure for $C$. As an example, consider the IFS maps

$$
w_{i}(x)=\frac{(-1)^{n}}{5} x+\frac{2}{5}\left\lceil\frac{i}{2}\right\rceil \quad \text { for } i=0,1,2,3,4,
$$

(where $\lceil x\rceil$ is the smallest integer greater than or equal to $x$ ) and the rotations $R_{i}$ where $R_{i}$ is a rotation in $\mathbb{R}^{2}$ of $(i-2) \pi / 4$ radians. The geometric attractor of the IFS is $[0,1]$ and the operator on vector-measures acts on measures with values in $\mathbb{R}^{2}$. (The IFS maps $w_{i}$ produce five contracted copies of $[0,1]$ with length $1 / 5$. The maps $i=0,2,4$ preserve the orientation whereas the maps $i=1,3$ reverse the orientation.) The first two iterations of the IFSVVM operator are illustrated in Figure 5. We use $p_{i}=1 /(\sqrt{2}+1)$ so that $\sum_{i} p_{i} R_{i}=I$. Since $s_{i}=1 / 5$, we have $\sum_{i} s_{i} p_{i}=p_{1}<1$.
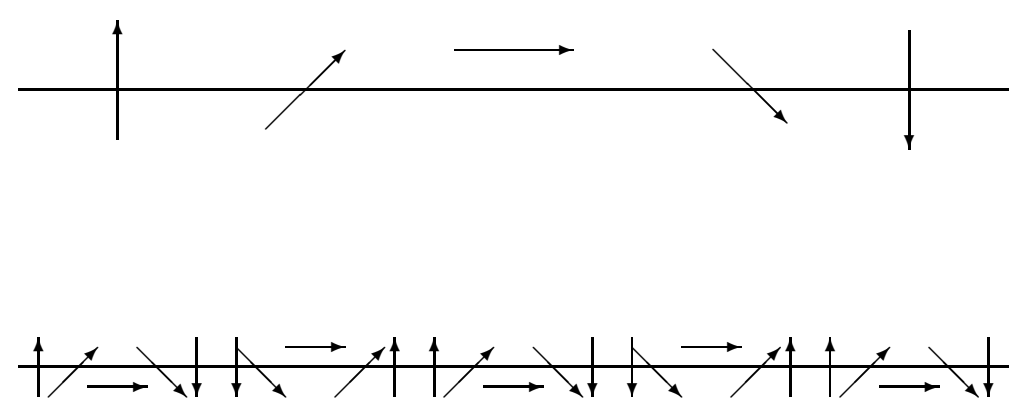

Figure 5: Iteration of the five-map IFSVVM in the text. The attractor is a fractal vector-measure on $[0,1]$

We also mention that for $T: \mathcal{S}_{0}\left(X, \mathbb{R}^{n}\right) \rightarrow \mathcal{S}_{0}\left(X, \mathbb{R}^{n}\right)$ the only possible fixed point is $\mu \equiv 0$, since $T$ is linear. This means that you cannot have one 
operator $T$ whose invariant vector measure is the tangent vector measure for a closed curve.

\subsection{Line integrals of vector fields over fractal curves}

Once again assuming that the curve $C \in \mathbb{R}^{2}$ is the attractor of an $N$-map IFS $w_{i}$ with probabilities $p_{i}$ and rotations $R_{i}$, the invariance of the vector measure $\mu=T \mu$ with support $C$ implies the relations

$$
\begin{aligned}
\int_{C} f d \mu & =\int_{C} f d(T \mu) \\
& =\int_{C}\left(T^{*} f\right) d \mu \\
& =\sum_{i} p_{i} \int_{C} R_{i}^{*} f\left(w_{i}(x)\right) d \mu .
\end{aligned}
$$

Whether or not $\mu$ is the tangent or normal vector to $C$ depends upon the choice of the vector $v$, as described earlier.

In the case of affine IFS maps $w_{i}$ and polynomial vector fields $f(x)$, the above relation yields a system of linear equations in the moments of the vector measure $\mu$ (specifically its components $\mu_{1}$ and $\mu_{2}$ ) that can be solved. These moments can then yield the value of the line integral $\int_{C} f d \mu$. Some sample calculations are presented in the Appendix.

\section{Green's Theorem for planar domains with frac- tal boundaries}

Suppose that we have a compact domain $D \subset \mathbb{R}^{2}$ whose boundary is the union of $M$ fractal curves $C_{i}$ each generated by an IFS. Suppose further that each curve $C_{i}$ has no self-intersections and that $C_{i}$ and $C_{j}$ only intersect at a point, and only if they are adjacent on the boundary of $D$. Finally, we suppose that the Lebesgue measure of each curve $C_{i}$ is zero.

Let $f: \mathbb{R}^{2} \rightarrow \mathbb{R}^{2}$ be a smooth function. For each $C_{i}$, we can compute

$$
\int_{C_{i}} f(x) \cdot d \mu_{i}(x)
$$

where $\mu_{i}$ is the tangent vector measure on $C_{i}$. Thus,

$$
\int_{\partial D} f(x) \cdot d \mu(x)=\sum_{i} \int_{C_{i}} f(x) \cdot d \mu_{i}(x)
$$

where we orient the curves $C_{i}$ in a consistent counter-clockwise manner.

Let $A_{i}$ and $B_{i}$ denote the initial point and endpoint, respectively, for each curve $C_{i}$ and let $v_{i}=\overrightarrow{A_{i} B_{i}}$. When we combine these vectors (or the line segments) together, we obtain a polygon $D_{1}$ as a first approximation to $D$. Applying the IFS of $C_{i}$ to $v_{i}$ yields a polygonal curve with endpoints $A_{i}$ and $B_{i}$. 
Combining these segments together yields a polygon $D_{2}$ as a second approximation to $D$. The boundary of $D_{2}, \partial D_{2}$, is an approximation to $\partial D$. Iterating this procedure yields a sequence of polygons $D_{n}$ that approximate $D$ along with the boundaries $\partial D_{n}$ that approximate $\partial D$. By construction, we know that $\partial D_{n} \rightarrow \partial D$ in the Hausdorff metric. Furthermore, we can use the IFS for $C_{i}$ to obtain an IFS operator $T_{i}$ for the tangent vector measure on $C_{i}$. Using the $T_{i}$ 's we obtain a sequence of vector measures $\mu_{n}$ such that $\mu_{n}$ is the tangent vector measure for $\partial D_{n}$.

Lemma 1 Let $\chi_{D}$ and $\chi_{D_{n}}$ be the characteristic functions of $D$ and $D_{n}$ respectively. Then $\chi_{D} \rightarrow \chi_{D_{n}}$ pointwise for almost all $x$.

Proof: $\quad$ By assumption, $\lambda(\partial D)=0$ so we only consider $x \notin \partial D$.

Suppose that $x \in \operatorname{int}(D)$. Let $\varepsilon=\operatorname{dist}(x, \partial D)$. Let $N$ be large enough so that for $n \geq N$ we have $h\left(\partial D_{n}, \partial D\right) \leq \varepsilon / 2$. Then $x \in \operatorname{int}\left(D_{n}\right)$ as well, so $\chi_{D_{n}}(x)=\chi_{D}(x)=1$.

Suppose that $x \notin D$. Again, let $\varepsilon=\operatorname{dist}(x, \partial D)$ and $N$ be large enough so that if $n \geq N$ we have $h\left(\partial D_{n}, \partial D\right) \leq \varepsilon / 2$. Then $x \notin D_{n}$ as well so $\chi_{D_{n}}(x)=$ $\chi_{D}(x)=0$.

Thus, $\chi_{D_{n}} \rightarrow \chi_{D}$ pointwise for almost every $x$.

This convergence is illustrated in Figure 6.
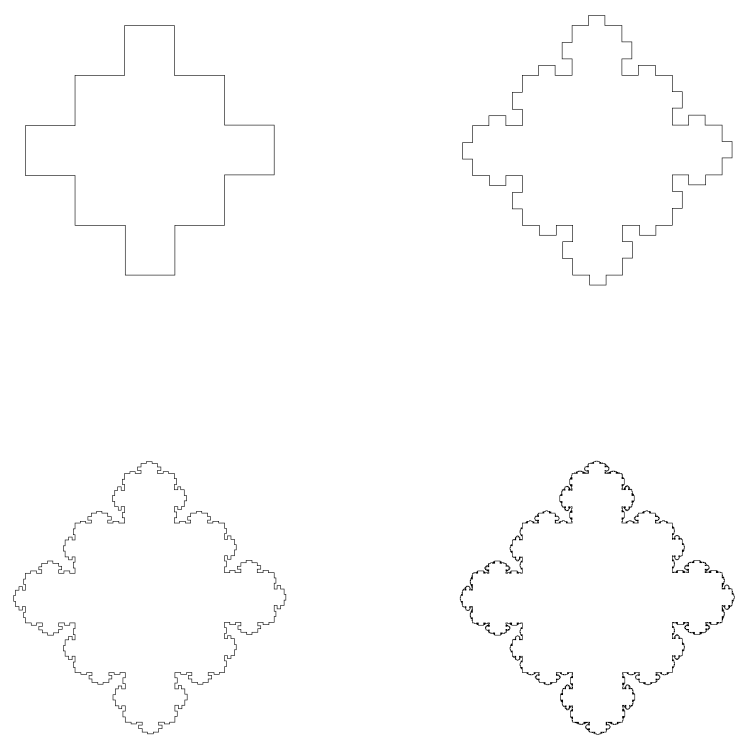

Figure 6: Illustration of $D_{n}$ and $D$ and their boundaries.

We are now in a position to prove Green's Theorem for fractal curves. 
Theorem 1 (Green's Theorem) Let $D \subset \mathbb{R}^{2}$ be a compact domain with $\partial D$ being the disjoint union of finitely many fractal curves and $\operatorname{dim}_{H}(\partial D)<2$. If $f$ is a smooth vector field, then

$$
\int_{\partial D} f(x) \cdot d \mu(x)=\int_{D}\left(\frac{\partial f_{2}}{\partial x}-\frac{\partial f_{1}}{\partial y}\right) d x d y
$$

where $\mu$ is the tangent vector measure to $\partial D$.

Proof: By our assumptions on $f$, we know that

$$
\chi_{D_{n}}\left(\frac{\partial f_{2}}{\partial x}-\frac{\partial f_{1}}{\partial y}\right)
$$

is a bounded sequence of functions which converges pointwise almost everywhere. Thus, by the Bounded Convergence Theorem,

$$
\int_{D_{n}}\left(\frac{\partial f_{2}}{\partial x}-\frac{\partial f_{1}}{\partial y}\right) d x d y \rightarrow \int_{D}\left(\frac{\partial f_{2}}{\partial x}-\frac{\partial f_{1}}{\partial y}\right) d x d y
$$

as $n \rightarrow \infty$. Furthermore, for each $n$, since $D_{n}$ is a polygonal set we have

$$
\int_{\partial D_{n}} f(x) \cdot d \mu_{n}(x)=\int_{D_{n}}\left(\frac{\partial f_{2}}{\partial x}-\frac{\partial f_{1}}{\partial y}\right) d x d y
$$

where $\mu_{n}$ is the tangent vector measure for $\partial D_{n}$.

Finally, we know that $\mu_{n} \rightarrow \mu$ by construction and the contractivity of the IFS operators $T_{i}$ and thus

$$
\int_{\partial D_{n}} f(x) \cdot d \mu_{n}(x) \rightarrow \int_{\partial D} f(x) \cdot d \mu(x)
$$

The same techniques allow us to prove the corresponding Divergence Theorem for the normal vector measure to the boundary of $D$, which we simply state below.

Theorem 2 (Divergence Theorem) Let $D \subset \mathbb{R}^{2}$ be a compact domain with $\partial D$ being the disjoint union of finitely many fractal curves and $\operatorname{dim}_{H}(\partial D)<1$. If $f$ is a smooth vector field, then

$$
\int_{\partial D} f(x) \cdot d \mu(x)=\int_{D}\left(\frac{\partial f_{1}}{\partial x}+\frac{\partial f_{2}}{\partial y}\right) d x d y
$$

where $\mu$ is the normal vector measure to $\partial D$. 


\section{Acknowledgments}

FM appreciates discussions with J. Anderson, who first suggested the idea of the "color chaos game". We also thank Dr. J. Levy-Vehel for helpful discussions and for drawing our attention to Ref. [10]. This research was supported in part by grants from the Natural Sciences and Engineering Research Council of Canada (NSERC) (ERV) and the National Science Foundation (FM).

\section{References}

[1] M.F. Barnsley, Fractals Everywhere, Academic Press, New York, 1988.

[2] M.F. Barnsley and S. Demko, Iterated function systems and the global construction of fractals, Proc. Roy. Soc. London, A399 243-275 (1985).

[3] K. Baron and A. Lasota, Markov operators on the space of vector measures; coloured fractals, Ann. Pol. Math. 69, 217-234 (1998).

[4] R. G. Bartle, A general bilinear vector integral, Studia Math., 15, 337-352 (1956).

[5] H. Brolin, Invariant sets under iteration of rational functions, Ark. Math. 6, 103-144 (1966).

[6] A. Bunde and S. Havlin (Eds.), Fractals and Disordered Systems, 2nd Edition (Springer Verlag, Berlin, 1996).

[7] J. Diestel and J.J. Uhl, Jr., Vector Measures, Mathematical Surveys 15, American Mathematical Society, Providence, RI, 1977.

[8] C.J.G. Evertsz and B.B. Mandelbrot, J. Phys. A: Math. Gen. 25, 1781 (1992).

[9] K.J. Falconer, The Geometry of Fractal Sets, Cambridge University Press, 1986.

[10] K.J. Falconer and T.C. O'Neil, Vector-valued multifractal measures, Proc. Royal Soc. London A452, 1-26 (1996).

[11] Y. Fisher (Ed.), Fractal Image Compression (Springer Verlag, NY, 1995); Y. Fisher (Ed.) Fractal Image Coding and Analysis, (Springer Verlag, Berlin, 1998); N. Lu, Fractal Image Coding, (Academic Press, NY, 1997).

[12] B. Forte, F. Mendivil and E.R. Vrscay, Chaos games for Iterated Function Systems with grey level maps, SIAM J. Math. Anal. 29, No. 4, pp. 878890 (1998).

[13] M. Giona, Contour integrals and vector calculus on fractal curves and interfaces, Chaos, Solitons and Fractals, 10, No. 8, pp. 1349-1370 (1999). 
[14] M. Giona and M. Giustiniani, Adsorption Kinetics on Fractal Surfaces, J. Phys. Chem. 100, 16690-16699 (1996).

[15] M. Giona, W.A. Schwalm, M.K. Schwalm and A. Adrover, Chem. Eng. Sci. 51, 4717-4729; 4731-4744; 5065-5076 (1996).

[16] J. Harrison, Flux across nonsmooth boundaries and fractal Gauss/Green/Stokes Theorems, J. Phys. A 32 no. 28, 5317-5327 (1999).

[17] J. Harrison, A. Norton, The Gauss-Green Theorem for fractal boundaries, Duke Math J. 67 no. 3, 575-588 (1992).

[18] J. Hutchinson, Fractals and self-similarity, Indiana Univ. J. Math. 30, 713-747 (1981).

[19] M. Keddam and H. Takenouti, C.R. Acad. Sci. Paris, Ser. II 302, 281 (1986).

[20] B. Mandelbrot, The Fractal Geometry of Nature, W.H. Freeman, San Francisco (1983).

[21] B. Sapoval, Phys. Rev. Lett. 73, 3314 (1994); Solid State Ionics 75, 269 (1995).

[22] B. Sapoval, Th. Gobron and A. Margolina, Phys. Rev. Lett. 67, 2974 (1991).

[23] B. Sapoval, R. Gutfraind, P Meakin, M. Keddam and H. Takenouti, Phys. Rev. E 48, 3333 (1993).

[24] W. Schwalm, B. Moritz, M. Giona and M. Schwalm, Phys. Rev. E 59, 1217 (1999).

[25] E.R. Vrscay, Iterated Function Systems: Theory, Application and the Inverse Problem, in Fractal Geometry and Analysis, NATO ASI Series C 346, Ed. J. Bélair and S. Dubuc (Kluwer, Dordrecht, The Netherlands, 1991), pp. 405-468.

\section{Appendix}

We now show how the invariance relation in Eq. (17) of the main text can be used to compute line integrals over IFS fractal curve attractors in the plane. We shall denote the components of relevant quantities as: $x=\left(x_{1}, x_{2}\right), f(x)=$ $\left(f_{1}\left(x_{1}, x_{2}\right), f_{2}\left(x_{1}, x_{2}\right)\right)$ and $\mu=\left(\mu_{1}, \mu_{2}\right)$. We are interested in computing the line integrals

$$
\int_{C} f(x) d \mu=\int_{C} f_{1}\left(x_{1}, x_{2}\right) d \mu_{1}+\int_{C} f_{2}\left(x_{1}, x_{2}\right) d \mu_{2},
$$


where $C$ is the attractor curve and $\mu$ is the IFS invariant measure, $\operatorname{supp}(\mu)=C$. With this understanding, the subscript $C$ will be dropped for the remainder of this discussion.

\section{Method 1: Recursive Computation of Moments}

We outline this method since it was discussed in [13]. Historically, moment recursion methods have been commonly used to compute integrals over IFS invariant measures $[2,25]$. We assume that the IFS maps are affine:

$$
w_{i}(x)=\mathbf{A}_{i} x+\mathbf{b}_{i}, \quad 1 \leq i \leq N,
$$

and that the linear operators $R_{i}$ in Eq. (7) are rotation operators defined by the angles $\theta_{i}$. In component form, the invariance relations become

$$
\begin{aligned}
& \int f_{1}\left(x_{1}, x_{2}\right) d \mu_{1} \\
& =\sum_{i} p_{i} \int f_{1}\left(a_{11, i} x_{1}+a_{12, i} x_{2}+b_{1, i}, a_{21, i} x_{1}+a_{22, i} x_{2}+b_{2, i}\right)\left(\cos \theta_{i} d \mu_{1}-\sin \theta_{i} d \mu_{2}\right) \\
& \int f_{2}\left(x_{1}, x_{2}\right) d \mu_{2} \\
& =\sum_{i} p_{i} \int f_{2}\left(a_{11, i} x_{1}+a_{12, i} x_{2}+b_{1, i}, a_{21, i} x_{1}+a_{22, i} x_{2}+b_{2, i}\right)\left(\sin \theta_{i} d \mu_{1}+\cos \theta_{i} d \mu_{2}\right) .
\end{aligned}
$$

If we now consider simple polynomial vector fields of the form $f_{1}\left(x_{1}, x_{2}\right)=$ $x_{1}^{i_{1}} x_{2}^{j_{1}}, f_{2}\left(x_{1}, x_{2}\right)=x_{1}^{i_{2}} x_{2}^{j_{2}}$, then the above relations yield a set of coupled equations that relate moments of the component measures $\mu_{i}$, to be denoted as

$$
g_{i j k}=\int x_{1}^{i} x_{2}^{j} d \mu_{k}
$$

However, such pairs of equations will generally be insufficient to determine the moments uniquely. The moments $g_{i j k}$ may be determined recursively, however, if they are computed in groups "from the bottom upwards" along diagonal sequences.

We begin the process with the selection of vector $v$ that defines our space $\mathcal{S}_{v}\left(X, \mathbb{R}^{n}\right)$ in Eq. $(6): \mu(X)=v$ implies that $\int d \mu=v$, so that

$$
g_{001}=\int d \mu_{1}=v_{1} \quad g_{002}=\int d \mu_{2}=v_{2}
$$

Now consider the following four cases for $f:\left(x_{1}, 0\right),\left(x_{2}, 0\right),\left(0, x_{1}\right)$ and $\left(0, x_{2}\right)$ to yield a system of four linear equations in the unknown moments $\mathbf{g}=\left(g_{101}\right.$, $\left.g_{011}, g_{102}, g_{012}\right)$. The structure of this system is

$$
\mathbf{C g}=g_{001} \mathbf{d}+g_{002} \mathbf{e}
$$


where the entries of the $4 \times 4$ matrix $\mathbf{C}$ and the $2 \times 2$ matrices $\mathbf{d}$ and $\mathbf{e}$ involve the IFS parameters.

This is the first step, $K=1$, of a recursive procedure in which the $(K+1)^{2}$ moments,

$$
g_{i j k}, \quad k \in\{1,2\}, i, j \geq 0, i+j=K,
$$

are computed in terms of lower order (i.e. lower $K$ ) moments. (This procedure is analogous to the computation of moments of two-dimensional IFS attractor sets [25].) The process is continued until all moments of the vector field used in Eq. (23) are computed.

As an example, we consider the von Koch curve of Figure 2. It is the attractor of the four-map IFS:

\begin{tabular}{cccccc}
$a_{11}$ & $a_{12}$ & $a_{21}$ & $a_{22}$ & $b_{1}$ & $b_{2}$ \\
\hline$\frac{1}{3}$ & 0 & 0 & $\frac{1}{3}$ & 0 & 0 \\
$\frac{1}{3}$ & $-\frac{\sqrt{3}}{6}$ & $\frac{\sqrt{3}}{6}$ & $\frac{1}{3}$ & $\frac{1}{3}$ & 0 \\
$\frac{1}{3}$ & $\frac{\sqrt{3}}{6}$ & $-\frac{\sqrt{3}}{6}$ & $\frac{1}{3}$ & $\frac{1}{2}$ & $\frac{\sqrt{3}}{6}$ \\
$\frac{1}{3}$ & 0 & 0 & $\frac{1}{3}$ & $\frac{2}{3}$ & 0
\end{tabular}

Since the endpoints of the curve are $A=(0,0)$ and $B=(1,0)$, we naturally set $v=(1,0)$. To compute the tangent vector we simply use the rotations $R_{i}$ that correspond to the IFS maps, i.e. $\theta_{1}=\theta_{4}=0, \theta_{2}=\frac{\pi}{3}, \theta_{3}=-\frac{\pi}{3}$. The probabilities are: $p_{i}=\frac{1}{3}$. The IFS operator $T$ associated with this IFSVVM, cf. Eq. (7), is contractive with contraction factor $c=\frac{4}{9}$, cf. Eq. (10).

The four moments for $K=1$ above are computed to be

$$
g_{101}=\frac{1}{2}, \quad g_{011}=\frac{\sqrt{3}}{20}, \quad g_{102}=-\frac{\sqrt{3}}{20}, \quad g_{012}=0 .
$$

To verify these calculations, consider the planar region $D$ enclosed by the von Koch curve $C$ (with endpoints $(0,0)$ and $(1,0))$ and the $x$-axis. Using geometric series, the area of $D$ is easily found to be $A=\frac{\sqrt{3}}{20}$. From Green's theorem, we may write $A=\int_{\partial D} f(x) d \mu$ where $f=\left(f_{1}, f_{2}\right)=\left(-x_{2}, 0\right), \mu$ is the tangent vector over $\partial D=[0,1] \cup C$ and the integration is oriented clockwise. The integration over $[0,1]$ yields zero. The integration over $C$ from $(1,0)$ to $(0,0)$ yields $(-1)^{2} g_{011}=\frac{\sqrt{3}}{20}$, in agreement with the above.

We now examine the divergence theorem applied to the planar region $D$ above. It will be necessary to compute line integrals involving the outer normal vector measure to the von Koch curve. If we choose the vector $v=(0,1)$, which is normal to the vector $\overrightarrow{A B}$, then the IFSVVM used above will place contracted copies of $v$ as normal vectors over the smaller line segments generated by the IFS maps, analogous to the tangent vector case in Figure 4. Moreover, these vectors will be outer normal vectors to the region $D$. In this case, the four moments for $K=1$ are

$$
g_{101}=\frac{\sqrt{3}}{20}, \quad g_{011}=0, \quad g_{102}=\frac{1}{2}, \quad g_{012}=\frac{\sqrt{3}}{20} .
$$


From the divergence theorem, $A=\int_{\partial D} f(x) d \mu$ where $f=\left(f_{1}, f_{2}\right)=\left(x_{1}, 0\right)$ and $\mu$ is the outer normal vector measure over $\partial D$. Once again, the integration over $[0,1]$ yields zero. The integration over $C$ (independent of direction) yields $A=g_{101}=\frac{\sqrt{3}}{20}$.

\section{Method 2: Direct Computation Using Adjoint Operator}

This method can be applied to a wider variety of problems that do not necessarily involve polynomial vector fields. The IFS maps also need not be affine. We make use of Eq. (16) in the main text. If we let the initial vector measure be $\mu_{0}=v \delta\left(x_{0}\right)$, where $\delta\left(x_{0}\right)$ denotes the Dirac unit mass at a point $x_{0}$, then

$$
\int f d \mu=\lim _{n \rightarrow \infty} S_{n}
$$

where

$$
S_{n}=\sum_{i_{1}, i_{2}, \ldots, i_{n}}^{N} p_{i_{1}} p_{i_{2}} \ldots p_{i_{n}} f\left(w_{i_{1}} \circ w_{i_{2}} \circ \ldots w_{i_{n}}\left(x_{0}\right)\right) R_{i_{1}} R_{i_{2}} \ldots R_{i_{n}} v .
$$

If $x_{0}$ is chosen to be a fixed point of one of the IFS maps, so that $x_{0} \in C$, then the sums $S_{n}$ involve the integration of $f(x)$ over $N^{n}$ point mass vector measures that lie on $C$. The computation of these sums can be performed recursively by enumerating an $N$-tree to $n$ generations.

We illustrate with the von Koch curve example. Let $v=(1,0)$ to produce the tangent measure to $C$. If $f=\left(x_{2}, 0\right)$, then $f d \mu=g_{011}=\sqrt{3} / 20$. The sums $S_{n}$ for $1 \leq n \leq 12$ along with the correct limiting value are listed below.

\begin{tabular}{cc}
$n$ & $S_{n}$ \\
\hline 1 & 0.0481137224 \\
2 & 0.0694971077 \\
3 & 0.0790005660 \\
4 & 0.0832241061 \\
5 & 0.0851010321 \\
6 & 0.0859350252 \\
7 & 0.0863054954 \\
8 & 0.0864699568 \\
9 & 0.0865428593 \\
10 & 0.0865750691 \\
11 & 0.0865891935 \\
12 & 0.0865952799 \\
\hline$g_{011}$ & 0.0866025404
\end{tabular}

